\title{
Noninvasive Epicardial and Endocardial Electrocardiographic Imaging of Scar-Related Ventricular Tachycardia
}

\author{
Linwei Wang ${ }^{1}$, Omar A. Gharbia ${ }^{1}$, Sandesh Ghimire ${ }^{1}$, B. Milan Horacek ${ }^{2}$, John L. Sapp ${ }^{2}$ \\ ${ }^{1}$ Rochester Institute of Technology, Rochester, NY, USA \\ ${ }^{2}$ Dalhousie University, Halifax, NS, Canada
}

\begin{abstract}
An effective treatment for scar-related ventricular tachycardia (VT) is to interrupt the circuit by catheter ablation. If activation sequence and entrainment mapping can be performed during sustained VT, the exit and isthmus of the circuit can often be identified. However, with invasive catheter mapping, only monomorphic VT that is hemodynamically stable can be mapped in this manner. A noninvasive approach to fast mapping of unstable VTs can potentially allow an improved identification of critical ablation sites. In this pilot study, noninvasive ECG-imaging were carried out on patients with unstable scar-related VT. The reconstructed reentry circuits correctly revealed both epicardial and endocardial origins of activation, consistent with locations of exit sites found during ablation procedures. The results also indicated that some reentry circuits involve both epicardial and endocardial layers, and can only be properly interpreted by mapping both layers.
\end{abstract}

\section{Introduction}

Sustained ventricular tachycardia (VT) are often due to a reentry circuit involving an area of ventricular scar [1]. Catheter ablation is an important therapeutic option for VT that requires an effective identification of myocardial sites critical to the reentry circuit. If the circuit can be mapped with a combination of activation and entrainment mapping, its exit and isthmus can often be identified [1]. However, with invasive catheter mapping, this is only feasible in a small percentage of VTs that are hemodynamically stable [1]. For the majority of ill-tolerated VTs or multiple VTs, the alternative of substrate mapping must be used during sinus or paced rhythm, which provides a less specific identification of circuit exits/isthmuses.

Noninvasive electrocardiographic (ECG) imaging uses high-density body-surface ECG data in combination with heart-torso tomographic scans to inversely calculate cardiac electrical activity [2]. Collectively, ECG-imaging techniques have demonstrated potential in a variety of clin- ical applications ranging from the mapping of atrial flutter and fibrillation [3] to the detection of myocardial ischemia [4]. For scar-related VT, there has been an interest in using ECG-imaging to electrically delineate the myocardial scar as an arrhythmogenic substrate for VT [5,6]. In addition, ECG-imaging has the unique ability to reveal the arrhythmia dynamics of VT. In the latter context, some studies have reported the use of ECG-imaging in reconstructing the activation isochrones and localizing the origin of VT $[7,8]$. These initial studies focused on VT mapping on only the epicardium of the heart and the reentry circuit was studied in the form of a static map of activation sequence. The temporal dynamics of the reentry circuit, its epicardial and endocardial involvement, and its relation to the myocardial scar substrates remain largely unexplored.

In the present study, we investigated the clinical potential of ECG-imaging in rapid mapping of unstable scarrelated VT. We hypothesized that: 1) simultaneous epicardial and endocardial reconstructions by ECG-imaging will improve the delineation of the 3D construct of the reentry circuit and its exit; and 2) the use of phase mapping [9] will enhance the visualization of the temporal dynamics of the reentry circuit. Experiments were conducted on patients who underwent catheter ablation of scar-related VT. The reconstructed reentry circuits were examined with respect to VT exits found during ablation procedures, as well as scar substrates identified from invasive mapping.

\section{Methods}

\subsection{Data Acquisition}

Patients were enrolled from those undergoing epicardial and endocardial catheter mapping and ablation of VT. As detailed in our previous study [7], axial CT was performed within 24 hours before the procedure. Body surface electrodes were applied immediately before the procedure according to the Dalhousie protocol [10], and 120-lead ECGs were recorded during induced VT.

The electrophysiology study and catheter ablation were performed according to usual clinical protocols that in- 
cluded venous and arterial vascular access with catheters placed at the His bundle region and in the right ventricle. LV endocardial mapping was performed by the retrograde aortic approach. The pericardial space was entered percutaneously using previously published techniques [11]. A 3D electroanatomic mapping system CARTO was utilized to acquire substrate maps during sinus or paced rhythm. VT was induced by programmed stimulation from the RV apex or outflow tract.

\section{2. $\quad$ ECG Imaging}

Off-line processing of 120-lead ECG recordings was performed using custom software. Faulty leads were manually identified and discarded. Noise was removed using wavelet filters. Baseline drift was corrected by fitting a low-order polynomial to the signal and subtracting it from the signal. Representative beats were manually selected for ECG imaging.

Patient-specific geometrical models of the heart and torso were created from axial CT scans using customwritten Matlab routines. The heart model included the epicardial and endocardial surfaces of both ventricles. The torso model was a homogeneous volume conductor model in the form of a triangulated body-surface mesh with 120 nodes corresponding to the surface electrodes.

The unknown variable was formulated as the extracellular potential on both epicardial and endocardial surfaces of the heart. These electrograms were calculated from the body-surface ECG at each time instant independently. As an initial feasibility study, a simple second-order Tikhonov regularization was used to obtain the inverse solution:

$$
\hat{\mathbf{s}}(\mathbf{t})=\arg \max _{\mathbf{s}(\mathbf{t})}\left\{\|\mathbf{H s}(\mathbf{t})-\phi(\mathbf{t})\|_{\mathbf{2}}^{\mathbf{2}}+\lambda\|\mathbf{L s}(\mathbf{t})\|_{\mathbf{2}}^{\mathbf{2}}\right\}
$$

where $\mathbf{s}(\mathbf{t})$ represents the unknown potential on the ventricular surface, $\phi(t)$ the ECG data on the body surface at the given time instant, and $\mathbf{H}$ the transfer matrix constructed for the subject-specific geometrical model. $\mathbf{L}$ is a Laplacian matrix on the ventricular surface. The first term of equation (1) seeks a solution that best fits the surface ECG data; the second term of equation (1) enforces a local smoothness of the spatial distribution of $\mathbf{s}(\mathbf{t})$. The regularization parameter $\lambda$ controls the relative importance of these two terms. In all the results reported in this paper, the value of was fixed at 0.5 . The computation time for reconstructing one complete beat of VT is on the order of $10^{-1}$ seconds. Therefore, in comparison to sequentially assembled electrograms at different locations of the heart over multiple beats, ECG-imaging simultaneously constructs all electrograms on the heart in a single beat in real time. It thus allows rapid mapping of unstable VTs.

To track the progression and spatiotemporal organization of reentry circuits during VT, we adopted the phase- mapping approach [9]. Phase mapping uses the Hilbert Transform to generate phase-shifted signals from the reconstructed electrograms, and then computes the instantaneous phase from these two signals for each node of the cardiac mesh at each time instant. The resulting maps track the progression of the phase from $-\pi$ to $\pi$ at each location of the cardiac mesh. The depolarization wave front (local activation time) is then defined as the time when the local phase crosses zero from negative to positive values.

\section{Results}

Two detailed VT reentry circuits reconstructed by ECGimaging on two patients are reported here.

\subsection{Epicardial reentry circuit}

The first VT was from a patient with non-ischemic cardiomyopathy. Sinus-rhythm substrate mapping revealed an infero-lateral scar located at the basal region of the LV epicardium (Fig.1A). During ablation, the VT exit was identified to be at the basal-lateral region of the LV epicardium.

In activation map (Fig.1B) reconstructed by ECGimaging, the exit (white star) is shown at the high basallateral region of the $\mathrm{LV}$ epicardium, consistent with the actual VT exit identified by contact mapping. The activation sweeps toward the apex at the anterior part of the heart, and rotates back toward the base at the inferior-lateral part of the epicardium. Slow conduction (crowding of isochrones) can be observed at the margin of the scar, as well as the basal-lateral region of the LV consistent with the location of a heterogeneous zone at the ablation sites.

To better appreciate the temporal dynamics of the reentry circuit, snapshots of phase maps of the reconstructed electrograms are shown in Fig.1C. The depolarization wave front (locations with phase $=0$ ) is tracked by the light blue contour and annotated by small white arrows. The timing of each snapshot with respect to the onset of the QRS is labeled and tracked on an ECG trace on lead V2 (i.e., $-20 \mathrm{~ms}=20 \mathrm{~ms}$ before the QRS onset). Lowvoltage regions identified from catheter maps (Fig.1A) are registered to the patient-specific ventricular model and displayed as a semi-transparent gray patch.

As shown, the reentry circuit exits at the high basallateral LV, which is consistent with the VT exit confirmed from the ablation procedure. In agreement with clinical knowledge, it is identified at $35 \mathrm{~ms}$ prior to the QRS onset during presystolic activation. The propagation was relatively slow before the QRS onset as the wave front approached the scar exit. After the QRS onset, the propagation shows a macro-rotation around the inferior lateral scar $(0-70 \mathrm{~ms})$ : note the relatively smooth propagation at the anterior LV where the extent of scar tissue is minimal, versus a notable delay of activation of the wave front at 


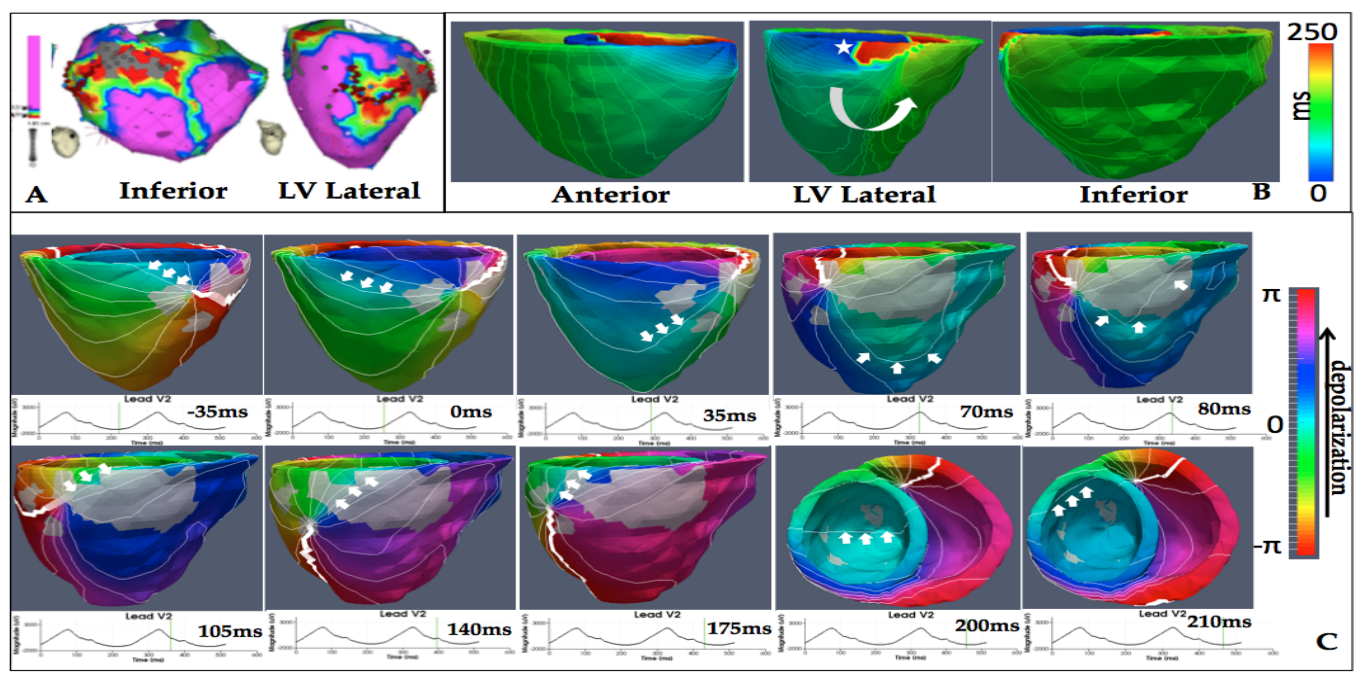

Figure 1. ECG-imaging results for VT1. Panel A: Bipolar voltage map of myocardial scar on the epicardium by catheter mapping. Panel B: Maps of activation time sequence re-constructed from 120-lead ECG during VT. Origin of activation is labeled by a white star at high basal lateral region of the LV epicardium, consistent with the exit site confirmed by ablation. A macro-reentry is seen counter-clock-wise anchored at lateral region of the LV epicardium. Panel C: Snapshots of the depolarization sequence of the reentry circuit reconstructed from 120-lead ECG.

mid-inferior LV at the site of the myocardial scar (70-80 $\mathrm{ms})$. After the circuit reenters the scar and before it exits, there is a long period (105-175 ms) when the activation wave front exhibits a zig-zag course of propagation inside the scar. This is followed by very slow conduction at the epicardium of the lateral LV throughout the diastolic phase of the activation, until the wave front exits again and repeats the next VT beat. Interestingly, as shown in the last two snapshots of the phase-map sequence, during the very-slow epicardial conduction before the next exit (occurring during the T-wave of the VT cycle), the same circuit also quickly depolarizes the endocardium of the LV with a sweep from inferior base down to apex and then up to the anterior base.

\subsection{Epicardial \& Endocardial Reentry}

The second VT came from a patient with prior infarction. As revealed by the bipolar contact signal amplitude (voltage) map, the myocardial scar is primarily located at inferior endocardium of the $\mathrm{LV}$ and extends somewhat to the inferior epicardium (Fig.2A). A large area of low voltage and unexcitable tissue (gray) was also found on the anterior surface of the epicardium, although it is not clear whether it corresponds to myocardial scar or fat layer. This clinical VT had an exit site at the high basal anterior region of the LV endocardium below the aortic valve.

ECG-based reconstruction of the activation sequence of this VT is shown in Fig.2B. From the epicardial view, the earliest epicardial breakthrough can be observed at high basal anterior LV consistent with an endocardial exit at the same location. However, the overall propagation pattern on the epicardium is suggestive of a focal rather than macro-reentrant arrhythmia. To appreciate the complete dynamics of this VT circuit, an understanding of endocardial involvement is necessary.

Fig.2C shows snapshots of the arrhythmia dynamics throughout the epicardial and endocardial layer. As shown, within $100 \mathrm{~ms}$ prior to the QRS onset of this VT, the exit of the circuit can be seen at the high basal region of the $\mathrm{LV}$ endocardium. After breaking through the epicardium, one part of the circuit (Fig.2C, row 2) sweeps through the basal-anterior region of the epicardium at a slower conduction velocity (0-30 ms, crowded isochrones), followed by a faster sweep through the rest of the anterior epicardium (30-90 ms) and rotates back to the basal epicardium of the RV (90-120 ms). At the same time, the other part of the circuit (Fig.2C, row 1) involves a counter-clock-wise macro-circuit across the lateral LV and anterior region of the epicardium, until it rotates back to the base of the RV epicardium (0-120 ms). These two waves meet, propagate through the endocardium of the RV (120-180 ms), the septum (180-240ms), and reenter the endocardium of the LV, until it is ready to exit again in the next VT beat. This epicardial and endocardial activation sequence suggests a stable figure-of-eight reentry circuit that exits at the basalanterior part of the LV, which can be appreciated in the endocardial view of activation sequence shown in Fig.2A. This can only be appreciated by examining its dynamics at both epicardial and endocardial layers. 


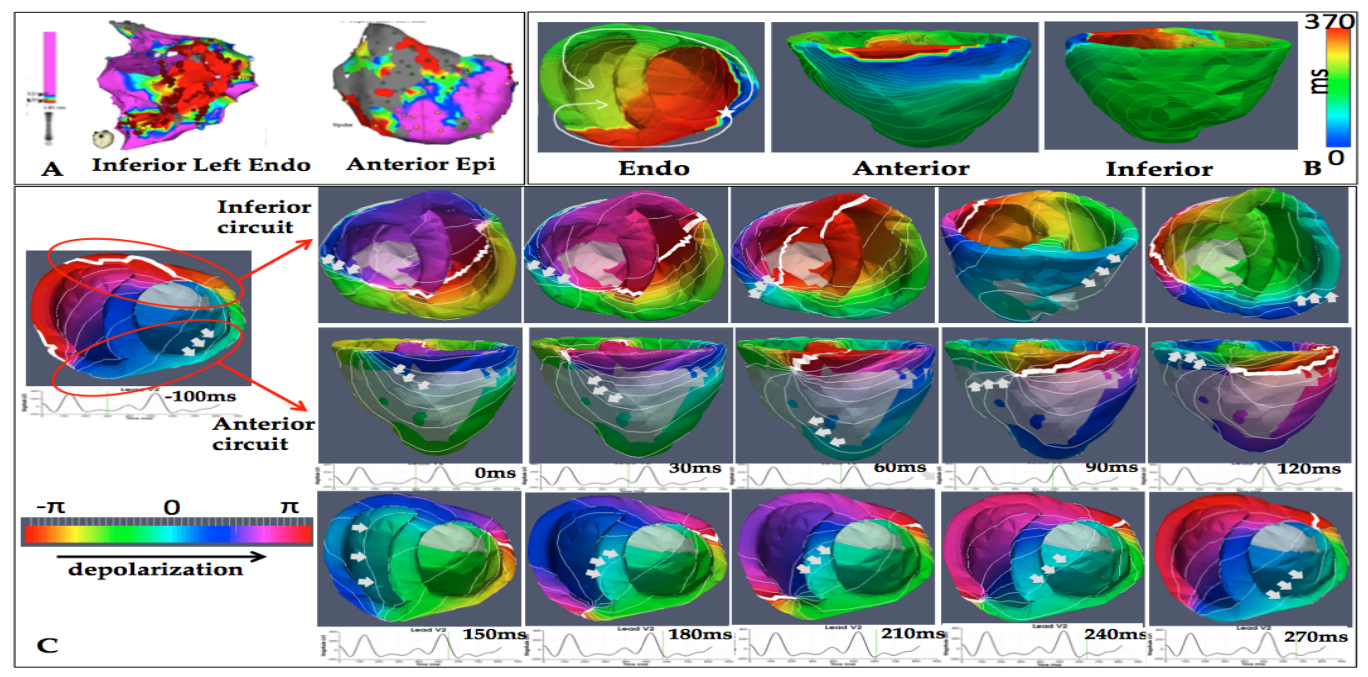

Figure 2. ECG-imaging results for VT2. Figures are presented as in Fig.1

\section{Conclusion \& Discussions}

This study demonstrated the feasibility of ECG-imaging in fast mapping of patient-specific reentry circuits for complex, unstable, scar-related VT. The example of VT 2 shows that, in reentry circuits that involve both epicardial and endocardial layers, only a complete epi-endo reconstruction can reveal the underlying dynamics of the circuit. Furthermore, by including endocardial reconstruction, we can differentiate between endocardial and epicardial exits, which may have important clinical implications for planning ablation treatment. In the future, a larger study is needed to establish the potential clinical use of ECGimaging in fast mapping of unstable VTs and eventually in guiding catheter ablation of VT.

\section{Acknowledgements}

This work was supported by the National Heart, Lung, and Blood Institute within the National Institutes of Health [grant number R21HL125998], the National Science Foundation [grant number CAREER ACI-1350374], the Canadian Institutes of Health Research, and the Heart $\&$ Stroke Foundation of Nova Scotia.

\section{References}

[1] Stevenson WG, Soejima K. Catheter ablation for ventricular tachycardia. Circulation 2007;115:2750-2760.

[2] Rudy Y, Messinger-Rapport B. The inverse problem of electrocardiography: solutions in terms of epicardial potentials. CRC Critical Reviews on Biomedical Engineering 1988;16:215-268.

[3] Haissaguerre M, Hocini M, Shah AJ, Derval N, Sacher
F, Jais P, Dubois R. Noninvasive panoramic mapping of human atrial fibrillation mechanisms: A feasibility report. Journal of Cardiovascular Electrophysiology 2013; 24(6):711-717.

[4] Wang Y, Cuculich P, Zhang J, Desouza KA, Vijayakumar R, Chen J, Faddis MN, Lindsay BD, Smith TW, Rudy Y. Noninvasive electroanatomic mapping of human ventricular arrhythmias with electrocardiographic imaging. Science Translation Medicine 2011;3(98):98ra84.

[5] Wang L, Dawoud F, Yeung S, an K. Wong PS, Lardo AC. Transmural imaging of ventricular electrophysiological activity and substrate in porcine hearts. IEEE Transactions on Medical Imaging 2013;32(4):731-747.

[6] Horacek BM, Wang L, Dawoud F, Xu J, Sapp JL. Noninvasive electrocardiographic imaging of chronic myocardial infarct scar. Journal of Electrocardiology 2015;in press.

[7] Sapp JL, Dawoud F, Clements JC, Horcek BM. Inverse solution mapping of epicardial potentials: quantitative comparison with epicardial contact mapping. Circulation Arrhythmia and Electrophysiology 2012;5(5):1001-1009.

[8] Wang Y, Cuculich PS, Zhang J, Desouza KA, Vijayakumar R, Chen J, Faddis MN, Lindsay BD, Smith TW, , Rudy Y. Noninvasive electroanatomic mapping of human ventricular arrhythmias with electrocardiographic imaging. Science Translational Medicine ;3(98):98ra84.

[9] Umapathy K, Nair K, Masse S, Krishnan S, Rogers J, Nash MP, Nanthakumar K. Mphase mapping of cardiac fibrillation. Circulation Arrhythmia ;3(1):105-114.

[10] Hubley-Kozey CL, Mitchell LB, Gardner MJ, Warren JW, Penney CJ, Smith ER, Horacek BM. Spatial features in body-surface potential maps can identify patients with a history of sustained ventricular tachycardia. Circulation 1995;92:1825-1838.

[11] Sosa E, Scanavacca M, d?Avila A. Gaining access to the pericardial space. The American Journal of Cardiology 2004;90(2):203-204. 